\title{
Health Management and Prognostics of Complex Structures and Systems \\ Guoyi Li
}

\author{
Arizona State University, Tempe, AZ, U.S. \\ guoyili@asu.edu
}

\begin{abstract}
This $\mathrm{Ph} . \mathrm{D}$. research focuses on developing reliable and effective structural health monitoring and prognostic health management frameworks for complex structures and system. The ultrasonic guided wave is used as a robust interrogation tool for structural level monitoring; numerical simulation, sensing architecture, signal processing and damage localization technique are being developed. For system level management, data mining techniques, with high-fidelity simulation platform, are used to establish a reliable fault detection and prediction for in-air aircrafts.
\end{abstract}

\section{PROBLEM STATEMENT}

Structural health monitoring (SHM) and prognostic health management (PHM) are emerging fields of current mechanical and aerospace engineering research. All structures and systems are operated under complex conditions such as excessive loading, fatigue, corrosion and impact; associated with the environmental effects and their inherent defects during manufacturing, such complex conditions are prone to resulting in unexpected structural and functional degradations, which may cause catastrophic failures. Aiming at securing the safety of structures and systems that are operated in critical environment, the SHM/PHM systems have been showing promising capabilities of detecting and localizing the structural damages or system anomalies, and providing quantitative information of remaining service life, time to maintenance as well as time to failure.

However, the modern structures and systems are expeditiously complicated because of the expectation of multi-function integration, strength-to-weight improvement, as well as weight and size reduction, which dramatically challenge the implementation of traditionally developed SHM and PHM technologies. Hence, it is an exponentially growing need for developing robust SHM and PHM methodologies to diagnose and predict the health state of such complex

Guoyi Li. This is an open-access article distributed under the terms of the Creative Commons Attribution 3.0 United States License, which permits unrestricted use, distribution, and reproduction in any medium, provided the original author and source are credited. structures and systems with their substantial implementation. Therefore, a multidisciplinary research is necessary for mechanical and aerospace engineering, leveraging signal processing and statistical technologies to develop robust health management and prognostic framework for complex structures and systems.

\section{EXPECTED CONTRIBUTIONS}

Contributions in structural level monitoring and system level health management are described as follows.

For structure level monitoring, ultrasonic guided wave (UGW) based techniques are promising for complex structures, due to their large inspection area and high sensitivity to small size damages. However, research and investigation are still in an urgent need to leverage the effectiveness of these techniques to monitor the health state of a complex structure. The primary reason is the UGW propagation in complex structure has not been well-understood and the complexities of the structures make the sensing signals even more complicated. Even high dimensional signal processing techniques showed robustness of detecting damages, they still have inherent limitations, including difficulties in localizing damages requirement of laser vibrometers, which limits its application in real-time SHM system. In order to assist the society to overcome such issues, current research will make following contributions to the structural level monitoring:

- Develop high fidelity numerical models for understanding wave propagation in composite materials, providing constructive guidance for sensor deployment and reducing experimental cost.

- Develop an effective damage localization methodology with a reference-free perspective that overcomes the difficulty of detection reflected waveform from the damage due to the high dissipation effect of structures such as foam core and honeycomb sandwich composite, while eliminating the need of baseline information.

- Develop an automatic and computationally efficient signal processing algorithms to enable the real-time monitoring capability of the developed framework; investigate performance of developed methodologies with multiple sensor architectures, excitations, and scales of the monitored structures. 
For system level monitoring, the proposed research will focus on the development of a real-time automated system health management framework for aircrafts. Inflight malfunction of aircraft subsystems may lead to lowered flight performances, hazardous conditions, and unexpected control issues; a fully integrated framework for safety monitoring of an in-air aircraft system is required; such framework is supposed to explicitly monitor the performance of each subsystem and accurately estimate performance of the complete aircraft system. Furthermore, sufficient computational efficiency is required for fulfilling the demand of onboard real-time monitoring to provide safety guidelines under faulty conditions at early stages. In order to serve the society to overcome such issues, current research will make following contributions to the system level PHM:

- Develop effective dimension reduction techniques to handle sensing dataset to eliminate unnecessary features, reduce the complexity in developing the detection models and avoid overfitting issues.

- Develop algorithm to enable the capability of onboard real-time monitoring of aircrafts and detect operational faults at an early stage providing pilots and control tower sufficient reactions time to the operational or structural anomalies.

- Develop a virtual real-time platform for algorithm validation and fault simulation, because it is impossible to obtain any possible aircraft faults in the real life. This research will be providing a simulation platform that can generate the data set with sufficient samples that fulfills the demand of model training, validating and testing.

\section{RESEARCH PLAN}

In order to efficiently and effectively develop robust SHM/PHM methodologies for complex structures and systems, the research plan of the proposed research is presented as follows.

Year One: In-depth literature review and technical learning of subjects proposed in the research. Develop a three-dimensional high fidelity numerical model of wave propagation in composite materials and validate the simulation results with the analytical elastic wave equations and the experiments performed in published literatures. The development and validation of the numerical wave propagation model, associated with the assessment of the presence of damages, will be presented in an international conference and submitted to a journal for publication within the $2^{\text {nd }}$ year.

Year Two: Design sensing architecture for detecting and localizing damages using UGW in structures with high attenuation effects, develop the detection and localization methodologies, and validate the designed sensor architectures and developed methods using in- house experiments. Then, study the effects of multiple types of sensors, excitation frequencies and damage modes on the detection accuracy. The development and validation of the localization methodology will be presented in an international conference and submitted to a journal for publication within the $3^{\text {rd }}$ year.

Year Three: Develop automatic and efficient signal processing techniques for sensing signal de-noising, dimensional reduction, feature space transformation and real-time UGW based damage localization. Simultaneously, by leveraging appropriate cutting-edge statistical techniques, such as machine learning and Bayesian inference, develop on-board real-time operational faults detection framework for in-air flight system monitoring. The development and validation of the signal processing algorithm will be presented in an international conference and submitted to a journal for publication within the $4^{\text {th }}$ year.

Year 4: Adopt the developed UGW based damage detection and localization techniques for the implementation on complex materials and structures with different specifications and damages scales. Meanwhile, develop a virtual platform for aircraft simulation with realistic faulty operations for the fault detection model visualization, validation and demonstration. The development of the model that combines the UGW based detection method and statistical method, and the PHM framework for aircraft system monitoring will be presented in two international conferences and submitted to two journals, respectively, for publication within one year.

\subsection{Work Performed}

A computationally efficient and generalized threedimension numerical model is developed to simulate wave propagation in anisotropic composite structures using computationally efficient scheme: Local Interaction Simulation Approach/Sharp Interface Method (LISA/SIM). The developed model accounts for electromechanical coupling between the host structure and the transducers. A demonstrative wave propagation contour in a quasi-isotropic carbon composite plate is shown in Figure 1. The attenuation and directivity of UGW are studied under varying composite layups, excitation frequencies, excitation modes, and material properties of composite panels and PZTs, and validated through published experimental results. With a fixed geometry and excitation frequency, the signal changes due to varying damage locations along the through-thickness direction are compared under both symmetrically and antisymmetrically collocated excitation architectures. Results and observations have been presented in IMECE 2016 and published in Journal of Intelligent Material Systems and Structures 2017. 


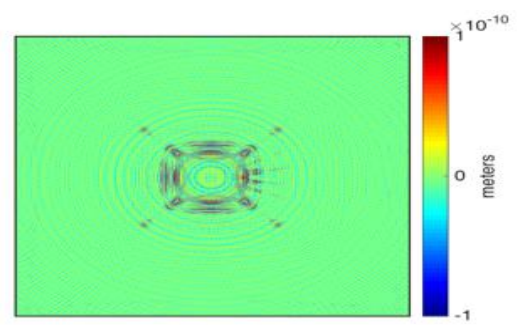

Figure 1. Simulated wave propagation in a quasi-isotropic carbon composite plate.

A real-time, converted guided wave based damage localization framework is developed for SHM of XCOR sandwich composites. Marco fiber composite (MFC) transducers and piezoelectric wafer active sensors (PWASs) are used to construct the sensing paths under a wide range of excitation frequencies, while the raw signals are de-noised through matching pursuit decomposition (MPD), a time-frequency based signal processing technique. Time-space representation is subsequently constructed by the envelopes of denoised signals, which are identified via a Hilbert transform. All possible wave mode trajectories are identified in the time-space domain; the number of wave modes containing in a particular signal series is characterized by a KDE; the source locations of all wave modes are successfully located through an iterative approach with a K-means clustering methodology. The developed framework, shown in Figure 2, is then experimentally validated through $\mathrm{X}$ COR panels with facesheet delamination and foam core separation. This framework is especially applicable when the damage induced reflected wave(s) are not detectable. The development of the novel signal processing and mode tracking approach has been published in the journal of Mechanical System and Signal Processing 2018 and will be presented in AHS 2018 in May; the development of the SHM framework for X-COR composite has been presented in IWSHM 2017 and submitted to the Structural Health Monitoring - An International Journal.

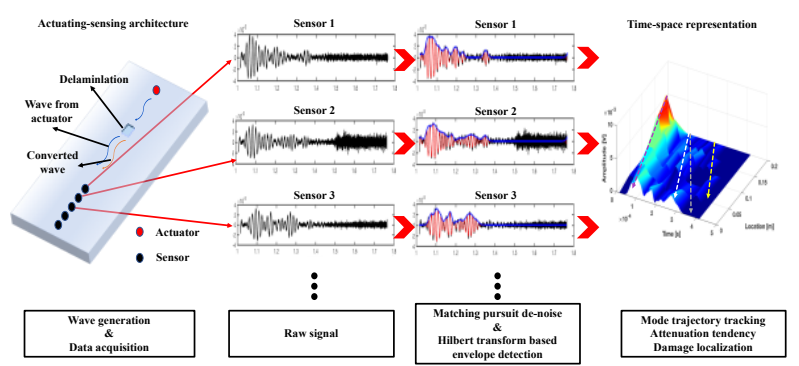

Figure 2. Developed SHM framework for damage localization in XCOR composite structures.

\subsection{Remaining Work}

Damage such as delamination at this interface between the integrated heat spreader (HIS) and epoxy sealant, of an integrated circuit (IC) package, can be caused due to manufacturing defects or service conditions, resulting in catastrophic failure of the package. Due to the robustness of structural interrogation as well as the developed signal processing and damage localization algorithms, the UGW based SHM framework for IC packages will be developed associated with advanced statistical models, to assess the health state of IC packages after manufacturing.

Then, the developed statistical model will be further extended for real-time health management of in-air aircraft of air traffic management system, aiming at detecting the operational faults at an early stage. A multivariate Gaussian mixture model (mGMM) has been developed as a real-time fault detector of the current proposed aircraft system, since the distribution of every attribute and correlation between attributes will be interpreted through training process. Then, a prognostic model will be developed to predict the future health state of the aircraft. In this research, historical real dataset of aircraft is investigated, and commercial flight simulator X-Plane is used to simulate the aircraft performance as a virtual health management platform for real time fault detection. A preliminary platform is shown in Figure 3; the details of the developed platform will be presented in PHM 2018.

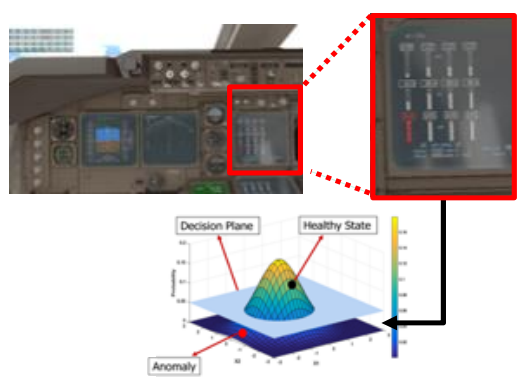

Figure 3. A virtual platform for aircraft monitoring.

\section{CONCLUSION}

A high-fidelity numerical simulation of UGW propagation in anisotropic and heterogenous materials has been developed, with the consideration of electromechanical coupling effect. Then, a robust SHM framework including an effective sensing architecture, signal processing method and mode tracking algorithm, has been developed for damage detection and localization in X-COR composites. In the following year(s), a SHM framework will be established for IC packages. Meanwhile, a PHM framework is being developed to monitor and predict the performance of aircrafts under in-air condition. Within one or two years, the proposed work will be completed, which would be beneficial to the PHM society by improving the accuracy and efficiency of current SHM/PHM methodologies, especially for complex structures and systems. 